\title{
El método geométrico en la física
}

Carlos Julio Luque Arias

Profesor Asociado

Departamento de matemáticas

Universidad Pedagógica Nacional

El nombre de la Geometría dejó de ser exacto desde que Euclides y sus contemporáneos dejaron de verla y estudiarla como una MEDIDA de la TIERRA, extendiendo su campo al estudio del ESPACIO como ente abstracto que contiene las cosas materiales y posee ciertas propiedades que hoy caracterizan al llamado ESPACIO EUCLIDIANO, entre ellas, el ser homogéneo (igual en todos sus puntos), isótropo (igual en todas sus direcciones), infinito e ilimitado (sin borde), 3-dimensional, no curvado.

Matemáticos y Físicos han ahondado en el concepto de espacio y medida hasta dejar el nombre GEOMETRIA sólo como un honroso recuerdo.

\section{EL ESPACIO FISICO:}

El primer espacio de la geometría fue la tierra (GEO); en principio limitada y supuestamente plana, pero ya suficiente para establecer la idea de distancia y con ella, empíricamente, algunos casos del Teorema de Pitágoras (Fig. 1) (Hindúes, Egipcios, Asirios) (1).

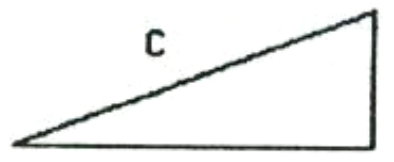

A
B

fig. 1

$$
C^{2}=A^{2}+B^{2}
$$

Con el estudio del espacio esférico centrado en la tierra del sistema Ptolomeico, fue posible el desarrollo de las ideas básicas de la Trigonometría (Aristarco, Hiparco, Ptolomeo).

La idea de espacio de Euclides prevaleció hasta influir decididamente en las primeras formulaciones matemáticas de la Física (2) (Keppler, Galileo, Newton) y en el estudio filosófico del espacio y el tiempo considerados como entes a-priori por Kant (3) implicando su unicidad e independencia del 
observador y por tanto impidiendo cualquier otra posibilidad de ser al espacio y al tiempo. El espacio «real» debía ser euclidiano.

A comienzos del Siglo $X X$ y a raíz de intentar la explicación de un experimento fallido (el de Michelson y Morley) fue necesario, como lo hizo ver Einstein, modificar nuestra concepción del espacio y el tiempo como entes absolutos, relativizar la simultaneidad, la distancia entre puntos del espacio y los intervalos de tiempo y con éllo sus consecuencias en lo que hoy se llama Teoría Especial de la Relatividad.

Fue Hermann Minkowski (4) quien mostró que si el tiempo se considera como una coordenada más y no como un parámetro, las fórmulas de la nueva Física (la relatividad) se podían escribir de una forma más familiar, sólo que en cuatro dimensiones.

$$
F^{u}=m_{0} \frac{d u^{u}}{d t} \quad u=1,2,3,4
$$

En lenguaje corriente significa: fuerza igual masa por aceleración (igual que antes). Las tres primeras componentes son ligeras modificaciones de la segunda ley de Newton; pero, ¿ y la cuarta? Resultó ser una famosa por sí sola:

Masa y energía son una sola cosa.

$$
E=m c^{2}
$$

Ya, en 1917 Einstein (5) formuló una teoría de la gravitación que generalizó la teoría especial de la relatividad, pero curvó el espacio-tiempo. Resultó que la fuerza de gravedad no existe, sino que las masas curvan el espaciotiempo (como cuando se mete el dedo en gelatina) y esa curvatura hace que las que eran rectas ahora sean curvas y ciertas curvas sean "rectas" (geodésicas) y por ejemplo la elipse que Marte describe en el espacio alrededor del sol es una «recta" en el espacio-tiempo curvo.

El salto de imaginación que se requiere para aceptar una cuarta dimensión es grande y difícil, pero después de la primera vez uno "pierde la vergüenza". Poco después, intentando formular una teoría que unificara la gravitación con el electromagnetismo, un maestro de escuela de apellido Kaluza, extendió a cinco las dimensiones del espacio-tiempo, pero no supo explicar dónde estaba esa quinta dimensión; por eso rápidamente pasó al olvido, hasta que Klein, con su prestigio lograra reavivar la discusión, afirmando que la quinta dimensión se compactificaba en un tamaño menor que el radio atómico; esto es que la quinta dimensión cierra al espaciotiempo en una especie de cilindro de radio muy pequeño y una altura de 
cuatro dimensiones que son las usuales del espacio-tiempo. Aún hoy (6) se discute la idea.

Después de esto fueron numerosos los avances en cuanto al número de dimensiones: por ejemplo, la Supersimetría (7): una teoría en la que se unifican Bosones o sea partículas con Spinm entero que obedecen la estadística de Bose-Einstein y no obedeciendo el principio de exclusión de Pauli, pueden compartir muchos el mismo estado (por ejemplo los fotones de la luz) y Fermiones o sea partículas que obedecen el principio de exclusión y no comparten su estado con ningún otro (por ejemplo los electrones y protones). En esta teoría el espacio tiene ocho dimensiones, en una de sus versiones. Más atrevida aún es la idea de las supercuerdas (8) que afirma que las partículas elementales no son puntuales sino cuerdas que vibran en espacios de diez ó de veintiséis dimensiones.

Pero el colmo del atrevimiento se debe a Hawking quien propuso que el espacio tiempo tiene dimensión fraccionaria (según la definición de Haussdorf) $4+\mathrm{e}$ donde e es un número pequeño. Yndurain y otros han probado que para que los datos experimentales sobre corrimiento Lamb coincidan con esta idea, e debe ser menor que $\left(10^{-7}\right)$ y Esto puede explicar ciertas fluctuaciones cuánticas del vacío y posiblemente tenga que ver con un procedimiento que se utiliza en Cromodinámica Cuántica, llamado regularización dimensional (9) y que consiste en calcular las integrales sobre un espacio de dimensión superior a cuatro $(4+\mathrm{e})$ cuyo caso convergen y luego tomar el límite cuando e tiende a 0.

Hoy existe una fuerte corriente iniciada Mandelbrot (10) en el estudio de estos espacios de dimensión fraccionaria, llamados fractales que no son ni líneas ni superficies, sino un intermedio entre éllas.

\section{EL ESPACIO MATEMATICO}

Desde el punto de vista matemático (sin querer ya ser "reales") el concepto de espacio ha ganado niveles de abstracción aún mayores, difíciles de comprender o por lo menos de imaginar.

En primera instancia el plano de Euclides, que se mantuvo plano por veinte siglos fue curvado por lobachevski (11) al modificar el postulado de las paralelas en un primer intento serio de estudiar espacios distintos al de Euclides. Claro que ya desde la antigüedad se conocía la geometría esférica, pero siempre vista inmersa en un espacio euclidiano.

Fue Gauss (12) quien propuso primero el estudio intrínseco de las superficies y Riemann quien generalizó el proceso a espacios de dimensión superior donde se puede definir una noción de distancia análoga al teorema de Pitágoras: 


$$
d s^{2}=g_{11}\left(d x_{1}\right)^{2}+g_{22}(d x 2)^{2}+\ldots .+g_{n n}\left(d x_{n}\right)^{2}
$$

y las $g_{i j}$ pueden depender de las coordenadas es decir, podemos medir la distancia entre dos puntos cercanos de manera diferente en diferentes regiones del espacio. Por ejemplo si $g_{11}=g_{22}=g_{33}=1$ y los demás son cero, el resultado es:

$$
d s^{2}=\left(d x_{1}\right)^{2}+\left(d x_{2}\right)+\left(d x_{3}\right)^{2}
$$

que corresponde al teorema de Pitágoras usual en tres dimensiones.

\section{Pero si:}

$g_{11}=1, g_{22}=g_{33}=g_{44}=-1$ y los demás son cero el nuevo "Teorema de Pitágoras", ahora en cuatro

dimensiones, es:

$d s^{2}=\left(d x_{1}\right)^{2}-\left(d x_{2}\right)^{2}-\left(d x_{3}\right)^{2}-\left(d x_{4}\right)^{2}$

y el espacio resultante es el de la relatividad especial llamado espacio de Minkowski.

Desde este punto de vista de Riemann (13), ya podemos hacer geometría sobre espacios de dimensión superior (por ahora entera y finita) y todo lo retorcidos que se quiera, siempre que digamos cómo se mide la distancia entre dos puntos cercanos. Por ejemplo, un espacio de colores o un espacio cuyos puntos sean las circunferencias o las rectas de otro espacio, y aquí el camino está abierto a la imaginación.

Hasta aquí hemos mantenido rectos los ejes de los sistemas de coordenadas que se usan para describir el espacio en cuestión, pero se puede, y de hecho se hizo, escoger curvas como ejes coordenados. Funciones como sen $(x)$ o un polinomio con algunas pocas condiciones (14) (continuidad, ortonormalidad) pero nuestra intuición ya empezó a temblar. El análisis de Fourier, el análisis espectral, el cálculo absoluto de Leví y Ricci, son hermosos ejemplos al respecto.

Se puede ser aún más atrevidos e inventar espacios donde cada punto es una curva de otro espacio y el resultado es un espacio de funciones de dimensión infinita contable o no contable (espacios de Hilbert, de Sobolev, de Wienner, de distribuciones) cuya dimensión es a veces tan grande, que no se pueden comparar con ninguna cosa conocida. (En aritmética ordinal transfinita llaman aleph 2 a este número) (16). 
Cabe naturalmente la pregunta: bueno, y esto, ¿para qué sirve?. Para un matemático basta que sea bello para que merezca su estudio (el concepto de "bello" lo decide él). Un físico no sólo exige la consistencia lógica y simplicidad que exige el matemático, sino que además exige que una teoría permita hacer predicciones que sean contrastables con el experimento y, por ende, generar tecnología. Sorprendentemente, estos monstruos matemáticos han permitido contruir física; por ejemplo, los espacios de Hilbert sirvieron de base a Dirac (17) para construir la mecánica cuántica y con ella el desarrollo de la microelectrónica con los avances de que hoy somos testigos.

Los espacios de funciones entraron por la puerta grande a la física de la mano de Feynmann (18) para construir la electrodinámica cuántica, la teoría más exacta que ha producido la mente humana (con diez cifras decimales de exactitud) y se quedaron para explicar cuantización, interacciones débiles y fuertes y generar en un futuro próximo tecnologías más avanzadas como microscopios bosónicos (con bosones vectoriales intermedios $Z_{0}$ ) mucho más potentes que los actuales microscopios electrónicos.

Hemos sobrevolado el concepto de espacio e intuido que ya está muy lejos del GEO primitivo, por tanto la geometría ya no es GEO. ¿será METRiA?.

\section{LA METIRICA}

La idea de distancia también ha tenido sus tropiezos y evoluciones hasta casi ya ni poder reconocerla.

La primera noción de distancia se la debemos a la experiencia directa y a su formalización en el teorema de Pitágoras que ya hemos mencionado. Una variación sencilla consiste en cambiar los coeficientes de $(\mathrm{dx})^{2}$. Es más fácil si juntamos todos los $g_{i j}$ en una matriz (por simplicidad hagámoslo en dos dimensiones).

Si $g_{i j}=\left[\begin{array}{c}10 \\ 01\end{array}\right] \begin{aligned} & \text { como ya se vió, la distancia se mide con el teorema de } \\ & \text { Pitágoras usual. }\end{aligned}$

Si $g_{i j}=\left[\begin{array}{l}10 \\ 00\end{array}\right]$ la distancia entre dos puntos cercanos es: 


$$
\begin{aligned}
& (d s)^{2}=\left(d x_{1}\right)^{2}+0\left(d x_{2}\right)^{2}=\left(d x_{1}\right)^{2} \\
& \text { o sea: } d s=|d x|=\left|x-x_{1}\right|
\end{aligned}
$$

Esto es, el valor absoluto de la diferencia de las abscisas.

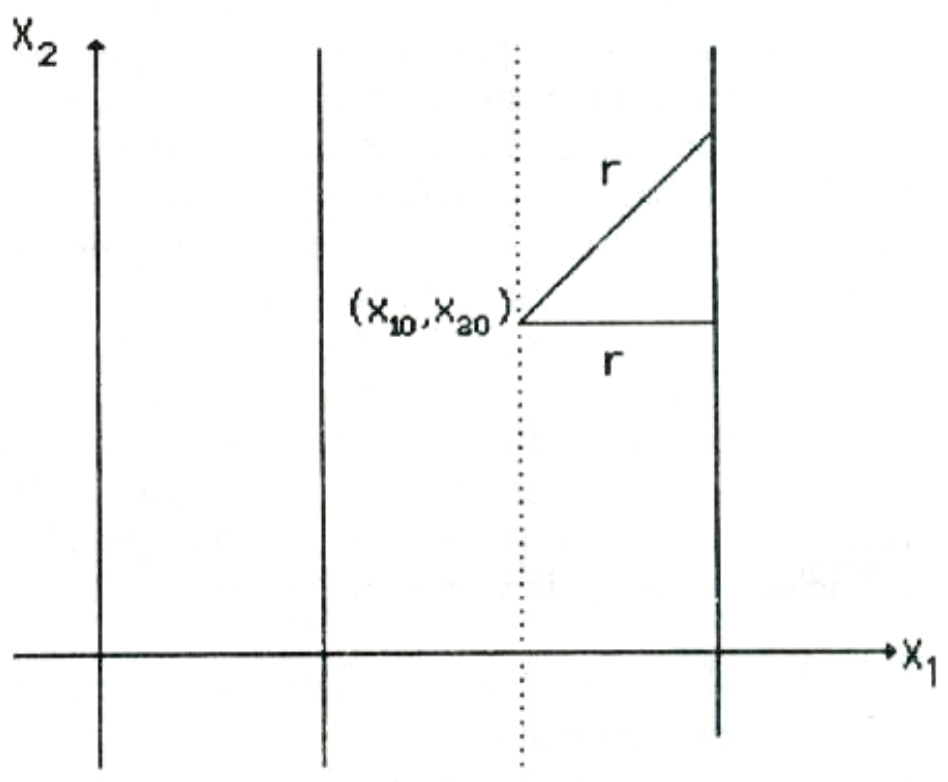

"circunferencias galileanas"

fiq. 2

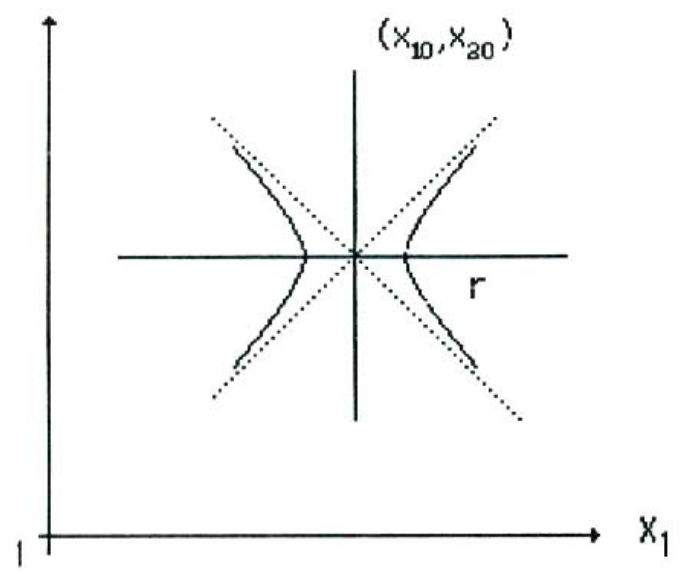

"circunferencias minkowskianas"

Fig. 3 
$Y$ una circunferencia es el conjunto de puntos $\left(\mathrm{x}_{1} \mathrm{x}_{2}\right)$ distancia a un centro, $\left(\mathrm{x}_{10}, \mathrm{x}_{20}\right)$ es constante, o sea:

$$
\left|x_{1}-x_{10}\right|=r
$$

que son dos rectas verticales.

Como se ve son unas circunferencias "extrañas"; abiertas y con infinitos centros (pues cualquier punto sobre la misma vertical del centro está a la misma distancia de los puntos de la "circunferencia". (Fig. 2).

Análogamente se pueden definir (19) rectas, ángulos, triángulos, etc. Curiosamente esta "extraña" geometría es la geometría del espacio-tiempo de Galileo, donde $x_{1}$ re presenta el tiempo y $x_{2}$ representa el espacio; una recta en este espacio representa un movimiento uniforme. El ángulo entre dos rectas es la velocidad relativa (teorema de adición de velocidades de Galileo) y a cada elemento de esta geometría galileana le corresponde un hecho de la cinemática clásica.

Escogiendo $\quad g_{i j}=\left[\begin{array}{c}10 \\ 0-1\end{array}\right] \begin{aligned} & \text { la "distancia" entre dos puntos } \\ & \text { cercanos es: }\end{aligned}$

$$
(d s)^{2}=\left(d x_{1}\right)^{2}-\left(d x_{2}\right)^{2}
$$

Las "circunferencias" son ahora hipérbolas equiláteras (Fíg. 3), los ángulos son hiperbólicos, etc.

Cada elemento y teorema de esta geometría (de Minkowski) tiene su interpretación en términos de cinemática relativista.

Hemos hecho cambios mínimos en $\boldsymbol{G}$ y los resultados ya son "extraños", si lo cambiamos más drásticamente los resultados pueden sorprendemos.

Por ejemplo, si:

O si en cambio:

$$
(d s)^{2}=R^{2}\left((d \beta)^{2}+\operatorname{sen}^{2} q(d \varnothing)^{2}\right)
$$

$r, \beta, \varnothing$ son las coordenadas esféricas y $\mathrm{R}$ es el radio de una esfera, obtenemos la geometría esférica; allí no hay paralelas, dos puntos no determinan una única recta, etc. 
O si en cambio:

$$
d s^{2}=R^{2}\left((d x)^{2}+\operatorname{senh}(x)(d \beta)^{2}\right)
$$

obtenemos la geometría sobre una pseudoesfera de Beltrami. Pero, ¿podemos ser absolutamente arbitrarios para escoger ds?.

Si sobre un espacio podemos definir ds de la forma

$$
(d s)^{2}=\sum g_{i j}(d x)^{\prime}(d x)^{j}
$$

el espacio se llama variedad Riemanniana y si estudio está ya bien adelantado (15).

Si debilitamos o fortalecemos las condiciones sobre ds obtenemos espacios métricos (20), variedades simplécticas (21) y muchos otros engendros.

Podemos, sin embargo, seguir otra ruta para hacer geometría, a partir de una idea de Klein plasmada e un programa presentado ante la Universidad de Erlangen y que consistía en usar la idea de grupo.

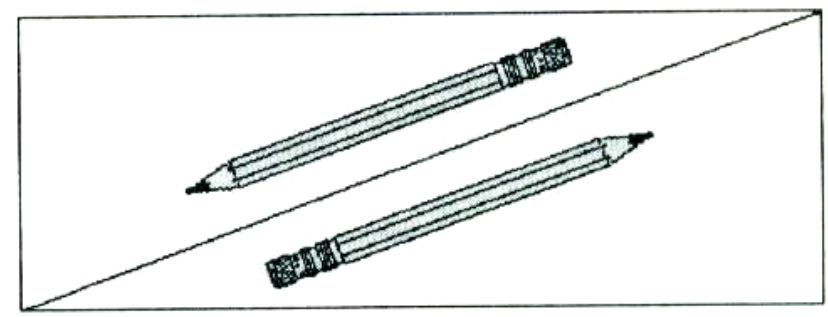

Euclides en sus elementos estableció que dos figuras geométricas son congruentes si al superponerse por un movimiento coinciden (claro que en geometría las figuras no se mueven). Pero no dijo explícitamente qué significaba movimiento, y de ahí se agarró Klein (23).

Digamos que tenemos un conjunto (cualquiera) y lo llamamos espacio, a cada subconjunto lo llamamos figura y definimos un conjunto de transformaciones (aplicaciones biyectivas) del Conjunto en sí mismo. Una de ellas es no transformar (aplicación idéntica), para que toda figura sea congruente consigo misma (si congruencia significa ser obtenido por un movimiento). Siempre que se tenga una manera de ir, se tenga una manera de regresar (transformación inversa) para que, si la figura uno es congruente a la dos, la dos sea congruente a la uno. Finalmente (o inicialmente), si hay una manera de ir de la figura uno a la figura dos y de la dos a la tres hay una 
manera de ir directamente de la uno a la tres; esto asegura la transitividad de la congruencia.

Así se estudian las propiedades de las figuras que no cambian bajo las transformaciones (que forman lo que los matemáticos llaman un grupo) y eso es la geometría del conjunto.

Por ejemplo, si tomamos como espacio el plano usual y como transformaciones las rotaciones, la distancia entre dos puntos no cambia y con ello todo lo que se pueda definir en términos de distancia como colinealidad, paralelismo, circunferencias, ángulos, etc., el resultado es la geometría de Euclides.

$\mathrm{Si}$ en cambio tomamos como transformaciones las proyecciones, la distancia entre dos puntos ya no es invariante, pero hay otras propiedades que sí (22); por ejemplo la razón doble de cuatro puntos colineales, el resultado es la geometría proyectiva.

Cuando el grupo es el de Fuchs obtenemos la geometría de Lobachevski y si es el de Galileo o Lorentz obtenemos la geometría galileana o minkowskiana que ya mencionamos.

Un ejemplo particularmente importante en la física moderna lo constituyen los grupos de matrices unitarias nxn con entradas complejas y determinante uno (abreviados $\mathrm{SU}_{\mathrm{n}}$ ) cuyas geometrías sobre espacios fibrados (24) producen las llamadas teorías de calibración (Gauge) (25) que describen las interacciones fundamentales: $U_{1}$ para el electromagnetismo, $\mathrm{SU}_{2}$ para las interacciones débiles, $\mathrm{SU}_{2}$ para las interacciones fuertes, $\mathrm{SU}_{5}$. para una teoría de campo unificado, etc.

Un último comentario lo merece la topología y el cálculo: si cambiamos las bolas usuales (circunferencias o esferas euclidianas) como base para la topología y en su lugar ponemos "circunferencias" galileanas o minkowskianas por ejemplo, la topología resultante no permitiría separar en circunferencias disyuntas puntos sobre una misma vertical (no sería Haussdorf) lo que quitaría la unicidad de los límites e impediría (así como está) una definición única de derivada o integral. Pero para eso están los matemáticos.

En resumen, la geometría ni es GEO, ni es METRIA. 


\section{BIBLIOGRAFIA}

(1) BOURBAKI, N.; Elementos de Historia de las Matemáticas. Alianza Universidad (1976).

(2) NEWTON, I; Principia Matemática.

(3) KANT, I; Crítica de la razón pura. Universales (1984).

(4) LORENTZ; MINKOWSKI; EINSTEIN; WEYL; The Principle of Relativity. Dover. (1952).

(5) BERGMAN; Introduction to the theory of Relativity. Dover. (1976)

(6) HERMANN, R; YANG-MILLS, KALUZA-KLEIN, Theories and the Einstein Program. Brookline. (1978)

(7) DE WITT; Supermanifolds. Cambridge. (1984)

(8) GREEN; SCHWARZ, WITTEN; Superstring Theory. Cambridge (1987)

(9) YNDURAIN; Quantum Cromodynamics. Springer. (1984)

(10)MANDELBROT, E; Fractals, form, chance, and dimension. Freemann (1977)

(11)KAGAN; Lobachevski. MIR (1978)

(12)STRUIK, D; Lectures on Classical Differential Geometry. Addison (1961)

(13) FOMENKO; DUBRODIN; NOVIKOV; Modern Gtometry. Springer (1984)

(14) LANG, S; Analysis, Dover, (1961)

(15) WEINBERG, 5; Gravitation

(16) SUPPES, P; Teoría axiomática de conjuntos. Norma (1974)

(17) DIRAC, P; Principios de la Mecánica Cuántica

(18) JAFFE; GLIMM; Quantum Physics. Springer (1987)

(19) YAGLOM, I; A simple non euclidian Geometry and its Physical basis. Springer (1979)

(20) MCARTY; Topology. Mac GrawHill (1976)

(21) ARNOLD; Mathematical Methods of Classical Mechanics. Springer (1976)

(22) COURANT; ROBI S; ¿Qué es la Matemática? Aguilar, (1974)

(23) EFIMOV; Geometria Superior. MIR (1984)

(24) NASH; SEN; Topology and Geometry for Physics. Academic. (1983)

(25) BAILIN; Gauge Theories (1982) 